\title{
A New System for Face Detection based on Eigen Face and Bat Algorithm
}

\author{
Nima ABEROMAND ${ }^{1 *}$, Rama Haghani PANAH ${ }^{2}$, Seyed Mahdi JAMEII ${ }^{3}$, \\ Bashir Bagheri NAKHJAVANLO ${ }^{4}$ \\ ${ }^{1}$ Department of Computer Engineering, Shahr-e-Qods Branch, Islamic Azad University, Tehran, Iran, \\ Nima.Aberomand@gmail.com \\ ${ }^{2}$ Department of Computer Engineering, Shahr-e-Qods Branch, Islamic Azad University, Tehran, Iran, \\ RamaHaghaniPanah@gmail.com \\ ${ }^{3}$ Department of Computer Engineering, Shahr-e-Qods Branch, Islamic Azad University, Tehran, Iran, \\ Jamei@Qodsiau.ac.ir \\ ${ }^{4}$ Member of Academic Islamic Azad University, Iran \\ *Corresponding Author: Nima Aberomand, Bagheri@iaufb.ac.ir
}

\begin{abstract}
Face detection consider image analysis and is one of the vital operations for people detection and face matching with existed data. Having enough information from an image is a method which examined in statistical and cognitive recognition which image processing and machine vision are tools of that. Image conception problem is related to have enough information from an image. In this approach, a new method for face detection proposed based on Eigen face as feature extraction and Bat algorithm as classification. This method used for finding people faces as vector in images. The obtained results show that proposed method has high accuracy and speed in comparison to other methods.
\end{abstract}

Keywords: Face Detection, Eigen face, Bat Algorithm, Image Processing, Machine Vision

\section{Introduction}

Face recognition and detection is the main problem in digital image processing and artificial intelligence which is important parts in today's technologies in relation to close-circuit security systems and photography. There are different commercial products such as photograph camera, image management applications, and social networks which use these systems. Face detection is the high level process in machine vision and cover a lot of technologies [1]. This subject can cover face analysis and recognition in images [2]. Generally, the main purpose of face detection divided into three main parts which contain face authentication, face recognition, and watch list [2]. The main point is that face detection and recognition are two different subjects. Actually, for recognizing faces, we need to detecting faces. Face recognition is the most important step in face detection. The most of the face recognition algorithm continue with identified position of faces in images [3]. 
One of the common method in object detection is considering a small frame in main image and detection will be are there any object in that window or not. If this method use, searching an algorithm is necessary to have ability to detect or not detect face in small frame with hundreds of pixels. Different elements can be efficient in face detection system such as training time, execution time, number of samples for training, and the ratio between detection and negative errors.

Existing methods for face recognition and detection divided into two parts, image-based and face-feature-based [4]. In image-based methods, different sets of facial image classify and for new face detection, this class use [4,5]. In face-feature-based methods, special feature of human faces used and face detection perform. As example, every face has two troughs in eyes area and something like a bow that are eyebrows put into troughs. Other samples are nose, lip, cheek, and etc [4].

There aren't any proposed method that have all of the best criterion. Each algorithm with improving one or more criteria, undermines others. It can be observed as a cases for existing challenges which are listed below:

- Not fixed some of elements of face such as hair, glass, mustache, and etc. that makes problem for recognition and detection.

- Lack of uniformity of luminance in different environment is one of the main problems.

- Stores image size difference and being different face ratio to the total size of image is one of the challenges before processing.

- Being different face angle in collected images is one of the inevitable challenges.

- Cover existing which may not visible a part of face due to face angle or located behind other objects.

In this approach, Eigen face used for feature extraction by considering Eigen-space and Eigenvalue. Among the reason of choosing Eigen face method for face detection in image are as follows:

- Independent from face geometry,

- Simple realization,

- The real-time feasibility realization even without having special hardware,

- Simplicity and quickly recognition in comparison to other methods,

- More success rate in comparison to other methods.

The challenges of Eigen face method is time calculating. If dataset is large, it takes time to recognizing person in image. In order to optimize processing time of Eigen face, Bat algorithm used in this approach. 


\section{Literature review}

There are a lot of research about face recognition and detection in images. In [6] a new framework proposed for recognizing faces by using $\mathrm{SOM}^{1}$ neural network and $\mathrm{KNN}^{2}$ method. This research is based on recent works such as Weighted Native Probabilistic Network [7], Quality Eigen Faces Techniques [8] and 2DPCA ${ }^{3}$ [9]. The proposed method show high performance with Kyrgyzstan people faces in comparison to others.

Another work used Adaptive Gabor Filter [10]. The proposed algorithm is based on EBGM [11] which recognized faces in stable condition and used normalization for reducing the diversity of images based on [12] that obtain eyes fixed coordinates with a geometric transformation and reduce image to $128 \times 128$ pixels.

In [13], another method use rectangular shapes and $\mathrm{SVM}^{4}$ for face detection. Using brightness and symmetry of eyes consider as resistant symptoms for finding pair of eyes. Three rectangular feature used as precision of work after SVM and then all of faces, detect on image. The proposed method is efficient and stable for eyeglass or optical changes in image. The datasets are CMU and BioID and results represent effectiveness of approach.

Another works tried to recognizing single or group of faces in image by using face segmentation and extraction [14]. The important parts of processing in this method are removing background, finding faces with skin color and movements of organs, geometric and appearance based, and edge detection. Image morphology for segmentation and filling holes in face and $\mathrm{GLCM}^{5}$ used for face recognition.

Gabor filter for feature extraction and $\mathrm{BP}^{6}$ neural network for training images due to face recognition used [15] and proposed method developed for performance providing as precision and retrieval accuracy.

Another method for face recognition as real-time processing used optical flow and histogram equalization [16] which experimental results confirm the power of face recognition.

In [17] face recognition and detection examined based on people skin color as single or group. PCA algorithm applied for correlation recognition of faces in images.

In [18] $\mathrm{RBF}^{7}$ neural network used for image classification due to face recognition. $\mathrm{LDA}^{8}$ algorithm used for feature extraction and RBF neural network for classification. This kinds of neural network use for reducing error numbers in classification which happens by nonlinear removable class. ORL dataset with 200 images used and result show the acceptable time and executable rate.

\footnotetext{
${ }^{1}$ Self-Organized Map

${ }^{2}$ K Nearest Neighbor

${ }^{3}$ Two Dimensional Principle Component Analysis

${ }^{4}$ Support Vector Machine

${ }^{5}$ Grey Level Co-occurrence Matrix

${ }^{6}$ Back Propagation

${ }^{7}$ Radial Basis Function

${ }^{8}$ Linear Discriminant Analysis
} 
In [19] random projection based partial feature extraction for recognizing robust faces proposed. Dimension reduction and increase used together. Dataset is FR and results show the robustness and time consuming process of face recognition.

\section{Suggested method}

\subsection{Pre-processing}

Fig. 1 shows the overall chart for face recognition that variety of methods proposed based on this framework [20].

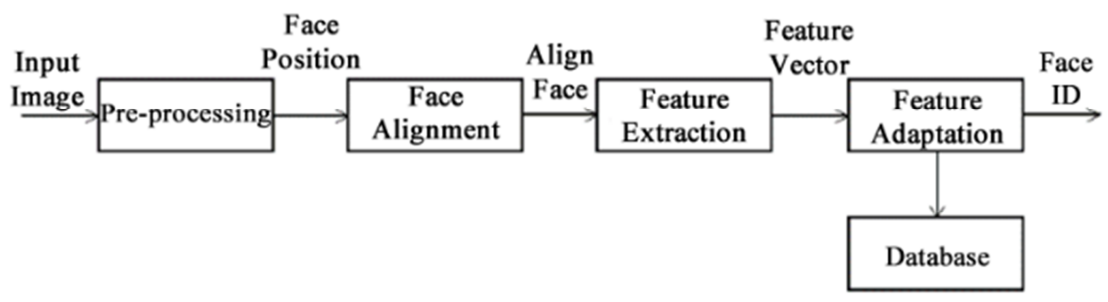

Fig. 1: Processing chart of face recognition

In pre-processing section, data are input images that should be normalized even for more performance optimization. After pre-processing, normalized facial image process in feature extraction for feature selection and then classification. Feature extraction divided into some features such as noise reduction, dimension reduction, dimension increase, and independent component separation. A lot of dimension reduction methods proposed until now. According to [20-25], branch and bound methods, sequential selection, common information, minimum redundancy with maximum communication and evolutionary algorithms approaches such as $\mathrm{PSO}^{9}, \mathrm{GAs}^{10}, \mathrm{ACO}^{11}$ and etc. proposed and compared. Among all of the dimension reduction algorithms, PCA have more popularity in comparison to others [26-28]. Eigen face is based on PCA which used for the first time in [29] that processed some images with faces and the best system vector has been handled for image compression. In another work [30] Eigen face used for face recognition in images.

\subsection{Eigen Face Method as Feature Extraction}

The main components of faces calculated in training set. Recognition operation formed by using face patterns in image space by Eigen face. One comparison build based on Euclidian Distance of Eigen-Vectors from Eigen face. If distance is small size, people face recognized in image. If distance is big enough, image considered as one of the belonging for one independent sample that trained by system. Eigen face flowchart shown in Fig. 2. [31].

\footnotetext{
${ }^{9}$ Particle Swarm Optimization (PSO)

${ }^{10}$ Genetic Algorithms (GAs)

${ }^{11}$ Ant Colony Optimization (ACO)
} 


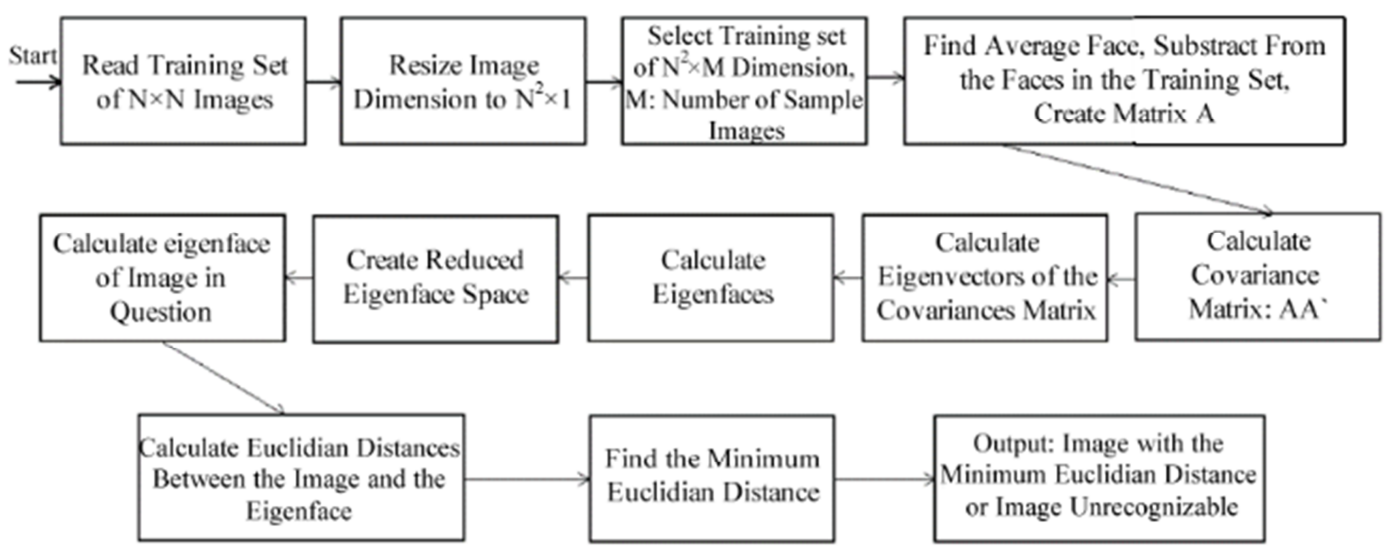

Fig. 2: Eigen face flowchart

As start point, training image read in $\mathrm{N} \times \mathrm{N}$ dimension and turn to $\mathrm{N}^{2} \times 1$ dimension. One training set build in $\mathrm{N}^{2} \times \mathrm{M}$ dimension that $\mathrm{M}$ is the number of sample image. Average of image set calculated from (1).

$\psi=\frac{1}{M} \sum_{i=1}^{M} \Gamma_{i}$

Based on (1), $\psi$ is average image, $\mathrm{M}$ is the number of images and $\Gamma_{i}$ is image vector. Corresponding Eigen faces with the highest Eigenvalues retain which these Eigen faces define face space. Eigen-space formed by image projection to image space which caused Eigen faces. So, vector weights are calculated. Image dimension tune for properties observation and image will be enhanced in pre-processing step. Then image weight vector and faces weight vector compare in image database. Then average face calculated and subtract from each existing image in training set. $A$ Matrix will be built by the results of subtraction operation. Comparison between each image and image average calculated as (2).

$\phi_{i}=\Gamma_{i}-\Psi, \quad i=1,2, \ldots, M$

In (2), $\phi_{i}$ is the difference between image and image average. Obtained matrix from subtraction operation or $A$ matrix, multiply in its transposed and finally $C$ covariance matrix form with (3).

$C=A^{T} A$

Based on (3), A matrix formed from difference between vectors. As an example it can be pointed out to $\mathrm{A}=\left[\phi_{1}, \phi_{2}, \phi_{3}, \ldots \phi_{\mathrm{M}}\right]$.C Matrix dimension is like $\mathrm{N} \times \mathrm{M}$. On the other hand, when $A$ level is equal to $\mathrm{M}$, only $\mathrm{M}$ from $\mathrm{N}$ number of Eigenvalue is equal to non-zero value. Then Eigenvalues of covariance matrix calculated and Eigen faces build by some trained images subtract to number of Eigenvalues class. The number of Eigen-vector classes are the total available faces in image. Selected sets of Eigen-vectors by $A$ matrix multiply due to dimension reduction of Eigen face. Eigen-vectors of smaller Eigenvalues match to smaller changes in covariance matrix. Other features of image maintain. The number of Eigen-vectors depends on 
image accuracy in database. Some of the selected Eigenvalues are Eigen faces. When Eigen faces obtained, images at database stored to Eigen face space and image weights in that projection of image space. Eigen-coefficients compared for define an image with Eigen-coefficient from image database. Then Eigen face build in image. Euclidian distance calculated between Eigen face of image and stored Eigen faces in previous step. Intended person recognize as a person that Euclidian distance is lower than threshold value in Eigen face database. If all of the Euclidian distance are bigger than threshold value, then there are no recognition of face in image and it will be overlooked. We try to extract features such as circular face region, eyes, and edge of cheeks. In all of the feature extraction if there are less features with the best result, it will show that the feature extraction was optimize and can be used in real.

\subsection{Bat Algorithm as Classification}

Up-to-date algorithm belonging to the family of evolutionary algorithms are principally inspired by nature and based on population intelligence.

Bat algorithm belongs to this group of algorithms designed by Yang in 2010 and is based on the features of reflection of bat voices [34] which used in this research as classification method for reducing time execution and optimize evaluation results. It this project, we assume that extracted features from Eigen face method are Bats and in any sentences when we write Bat, it means that extracted features.

Small bats usually use echolocation for detection of prey, avoiding the obstacles, and finding shelter to stay during night in darkness [33].

Once the sound velocity in the air revolves around $v=340 \mathrm{~m} / \mathrm{s}$, the ultrasound $\lambda$ wavelength with a constant frequency of $\mathrm{f}$ which is $\lambda=\mathrm{v} / \mathrm{f}$, ranges between 2-14 $\mathrm{mm}$ for a normal frequency range, i.e. $25-150 \mathrm{KHz}$.

Each bat with a velocity of $v_{i}^{t}$ and position of $x_{i}^{t}$ in repetition period of $t$ participates in a search space or a d-dimensional solution. Among all bats, there is a series of $X_{*}$ solution, which is the best current state. Therefore, the three mentioned rules in [34] culminate in development of the (4), (5), and (6) for $x_{i}^{t}$ and $v_{i}^{t}$ which try to classify the extracted features.

$\mathrm{f}_{\mathrm{i}}=\mathrm{f}_{\min }+\left(\mathrm{f}_{\max }-\mathrm{f}_{\min }\right) \beta$

$v_{i}^{t}=v_{i}^{t}+\left(x_{i}^{t-1}-x_{*}\right) f_{i}$

$x_{i}^{t}=x_{i}^{t-1}+v_{i}^{t}$

Where, $\beta \epsilon[0,1]$ is a random vector taken from a homogenous distribution. As mentioned previously, it is possible to use wavelengths or frequencies of implementation, where using $f_{\min =0}$ and $f_{\max }=0(1)$, dependent on the range size, the intended program is formulated. First, a uniform frequency generated between $\left[\mathrm{f}_{\min }, \mathrm{f}_{\max }\right]$ is assigned to each bat randomly. For this reason, the bat algorithm can be utilized as an algorithm for adjustment of frequency to present a balanced exploration and exploitation combination. The sound volume and pulse propagation rate 
can produce optimal solutions by developing a mechanism for automatic controlling and targeting within an area.

In order to provide an effective mechanism for controlling the exploration and exploitation and, if required, directly converting to the exploitation phase, The changes in volume $A_{i}$ and the propagated pulse rate $r_{j}$ during repetitions is obtained. Since sound volume of a bat usually declines once, a bat tries to find a prey that are faces in image, whereas when the pulse propagation rate grows, the sound volume can be considered between $A_{\min }$ and $A_{\max }$ with the assumption of $A_{\min }=0$ as any value this is simpler. This means that a bat only finds the prey and has stopped the sound propagation temporarily. With these assumptions, we have (7).

$A_{i}^{t+1}=\alpha A_{i}^{t}, \quad r_{i}^{t+1}=r_{1}^{0}[1-\exp (-\gamma t)]$

Where, $\gamma$ and $\alpha$ are the constants. Indeed, $\alpha$ bears a close resemblance to the cooling criterion in the simulated cooling algorithm [34]. For each $0<\alpha<1$ and $0>\gamma$, (8) exists.

$\mathrm{A}_{\mathrm{i}}^{\mathrm{t}} \rightarrow 0, \quad \mathrm{r}_{\mathrm{i}}^{\mathrm{t}} \rightarrow \mathrm{r}_{1}^{0}, \quad$ as $\mathrm{t} \rightarrow \infty$

In the simplest form, one can consider $\alpha=\gamma$ and use the $\alpha=\gamma=0.9$ up to 0.98 in the simulations. In order to improve the variety among the possible solutions, in [32] a method has been presented for random motion. First, a solution is selected from among the best solutions. Then, a new solution for every bat that has accepted the condition of random motion $>r_{i}$, will follow (9).

$\mathrm{x}_{\text {new }}=\mathrm{x}_{\text {old }}+\varepsilon \overline{\mathrm{A}}(\mathrm{t})$

Where $\bar{A}(t)$ is the mean sound volume of all is bats at the time of $t$ and $\varepsilon \in[-1.1]$ is the attempt for straitening and the random motion power. It means that when there is no face in images and Eigen face can't find any features of faces in image, Bat algorithm try to work in parallel to find any features. Actually it combines with Eigen values and Eigen-vectors of Eigen face to find any features to classify and then detect a face. For each algorithm repetition, the sound volume $A_{i}$ and pulse propagation rate of $r_{j}$ are upgraded in the form of (10) and (11).

$\mathrm{A}_{\mathrm{i}}(\mathrm{t}+1)=\alpha \mathrm{A}_{\mathrm{i}}(\mathrm{t})$

$\mathrm{r}_{\mathrm{i}}(\mathrm{t}+1)=\mathrm{r}_{\mathrm{i}}(0)[1-\exp (\gamma \mathrm{t})]$

Sound volume means combination with Eigen face method and pulse propagation rate means finding features based on Eigenvalues and Eigen-vectors of Eigen face. Each bat position in the search space deals with the encoding of a set of features that shows. Therefore, for each of them, the bat is trained in $Z_{1}$ and evaluated in $Z_{2}$ for the fitting value of each bat. Note that the set of training and evaluation in bats might be variable once each of them encodes a set of features. First, the initial population of bats is considered. Next, the position of bats is specified with the value of the random selection that examines whether the feature has been selected or not. Thereafter, the new set of training and evaluation is initiated with the selected features, followed by upgrading of the fitting value for each bat. In addition, the volume $A_{i}$ and pulse propagation 
rate of $r_{j}$ is upgraded provided that the new solution is accepted. The maximum output of the function is considered as an index and the bat fitting value results in the maximization of the fitting function. The position and rate should be upgraded, where it is carried out given the maximum value obtained for the fitting function for each bat.

\section{Simulation results}

In order to evaluate result of classification, some methods used such as accuracy, sensitivity, specificity, $\mathrm{MSE}^{12}, \mathrm{SNR}^{13}$, and $\mathrm{PSNR}^{14}$. Equation (12) show the accuracy formula.

Accuracy $=100 \times \frac{\mathrm{TP}+\mathrm{TN}}{\mathrm{TN}+\mathrm{TP}+\mathrm{FN}+\mathrm{FP}}$

In (12), TP means True Positive, TN is True Negative, FP is False Positive, and FN is False Negative. Equation (13) show the sensitivity formula and (14) show the specificity.

Sensitivity $=\frac{\mathrm{TP}}{\mathrm{TP}+\mathrm{FN}}$

Specificity $=\frac{\mathrm{TN}}{\mathrm{TN}+\mathrm{FP}}$

SNR formula show in (15), (16) for power signal and (17) for noise signal.

$\mathrm{SNR}=\frac{\mathrm{P}_{\text {signal }}}{\mathrm{P}_{\text {noise }}}$

$\mathrm{P}_{\text {signal }, \mathrm{dB}}=10 \log _{10}\left(\mathrm{P}_{\text {signal }}\right)$

$\mathrm{P}_{\text {noise }, \mathrm{dB}}=10 \log _{10}\left(\mathrm{P}_{\text {noise }}\right)$

PSNR based on MSE show in (18).

PSNR $=10 \cdot \log _{10}\left(\frac{\mathrm{MAX}_{\mathrm{I}}^{2}}{\mathrm{MSE}}\right)$

In (18), $\mathrm{MAX}_{I}^{2}$ is the maximum amount of signal. Table 1 shows the obtained value of each evaluation methods in classification time which obtained by 100 input image and average between them.

TABLE 1: Obtained results of classification

\begin{tabular}{|c|c|}
\hline Accuracy & $96.81 \%$ \\
\hline Sensitivity & 86.3959 \\
\hline Specificity & $93.9742 \%$ \\
\hline MSE & 0.51 \\
\hline SNR & $32.159 \mathrm{~dB}$ \\
\hline PSNR & $15.921 \mathrm{~dB}$ \\
\hline
\end{tabular}

\footnotetext{
${ }^{12}$ Mean Square Error

${ }^{13}$ Signal to Noise Ratio

${ }^{14}$ Peak Signal to Noise Ratio
} 
Bulletin de la Société Royale des Sciences de Liège, Vol. 86, special edition, 2017, p. 461 - 473

Table 2 shows the comparisons between some methods and proposed.

TABLE 2: Comparison of recent methods and proposed one

\begin{tabular}{|c|c|c|}
\hline Method & No. of Image in Training & Accuracy \\
\hline PCA [1] & 400 & $79.65 \%$ \\
\hline PCA based on $\mathrm{ICA}^{15}[1]$ & 400 & $92.34 \%$ \\
\hline $\mathrm{ICA}^{16}[2]$ & 170 & $81.35 \%$ \\
\hline HMM $^{17}[3]$ & 200 & $84.00 \%$ \\
\hline Active Shape Model [4,5] & 100 & $78.12 \%-92.05 \%$ \\
\hline Wavelet Transform [6] & 100 & $80.01 \%-92.10 \%$ \\
\hline SVM $[7,8]$ & 100 & $85.09 \%-92.11 \%$ \\
\hline MLPNN $^{18}[9]$ & 100 & $93.70 \%$ \\
\hline Other Eigen face methods [10] & 100 & $95.00 \%$ \\
\hline Proposed Method & 100 & $96.81 \%$ \\
\hline
\end{tabular}

As shown in table 2, our proposed method has the better accuracy rate in comparison to others with $98.81 \%$ with 100 training image. Results of the table 2 are shown in Fig. 3.

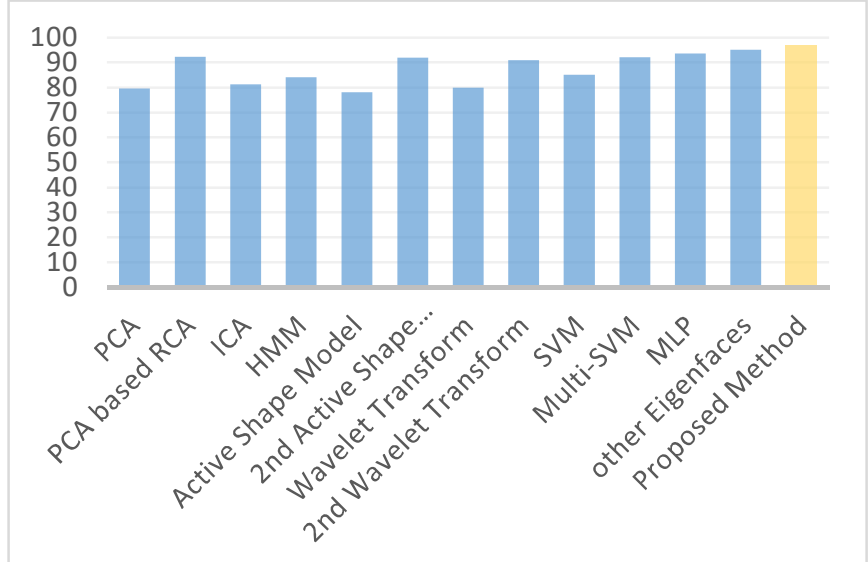

Fig. 3: Comparison chart of recent methods and proposed method

\footnotetext{
${ }^{15}$ Relevant Component Analysis

${ }^{16}$ Independent Component Analysis

${ }^{17}$ Hidden Markov Model

${ }^{18}$ Multi-Layered Perceptron Neural Network
} 
This proposed method can be test on other datasets i.e. UMIST, Yale, PurDueU, XM2VTS, BioID, MITCBCL, ORL, PIE, IMM, CMU, Face Champions, JAFEE, and Face Sketch due to stability and state changes of this approach. Some image tested and shown in Fig. 4.
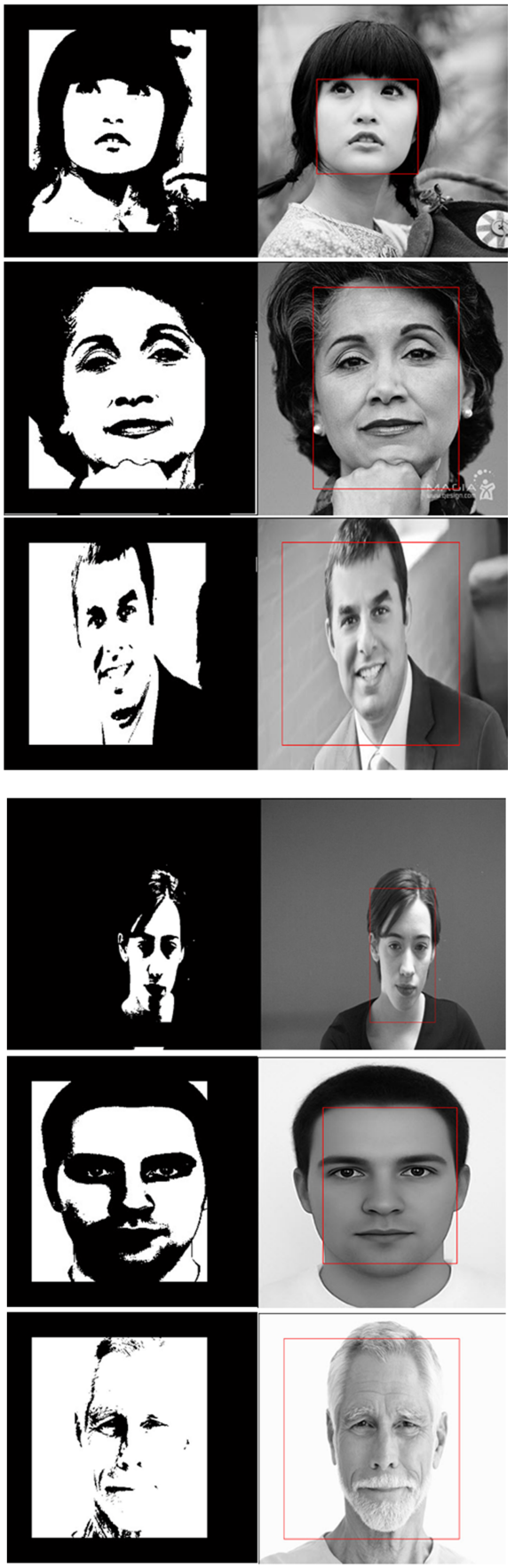

Fig. 4: Results of face detection in images 


\section{Conclusion}

In this research, a new method for face detecting in images proposed. Recognition operation of faces in image can be used for future process of face detection and matching that have several application in other sciences. The proposed method is based on Eigen face for feature extraction and Bat algorithm for classification. Of course, there is a combination of these two methods when Eigen face can't find any features, Bat algorithm try to find features. The features are circular face region, eyes, and edge of cheeks. The proposed approach show that have better performance in comparison to recent methods and evaluations i.e. accuracy, sensitivity, specificity, MSE, SNR, and PSNR proved the algorithm.

\section{References}

[1] Shradha Tiwari, Prof. J. N. Chourasia and Dr. Vijay S. Chourasia. "A Review of Advancements in Biometric Systems." International Journal of Innovative Research in Advanced Engineering, Vol. 2, Issue 1, 2015.

[2] Talele K. T. and Sunil Kadam. "Face detection and geometric face normalization." 10th IEEE Region Conference of TENCON, 2009.

[3] Hsu Rein-Lien, Mohamed Abdel-Mottaleb and Anil K. Jain. "Face detection in color images." IEEE Transactions on Pattern Analysis and Machine Intelligence, Vol. 24, No. 5, pp. 696-706, 2002.

[4] Lang Liying and Weiwei Gu. "Study of face detection algorithm for real-time face detection system." Second IEEE International Symposium on Electronic Commerce and Security (ISECS), Vol. 2, 2009.

[5] Zakaria, Zulhadi and Shahrel Suandi. "Face detection using combination of Neural Network and Adaboost." 10th IEEE Region Conference of TENCON, 2011.

[6] Nazir Ali Shoukat. "A Framework for Face Recognition using Self Organizing Map (SOM) and Soft k-NN Ensemble." International Journal of Application or Innovation in Engineering \& Management (IJAIEM), Vol. 1, Issue 1, 2012.

[7] A. M. Martinez. "Recognizing imprecisely localized, partially occluded, and expression variant faces from a single sample per class.” IEEE Trans. Pattern Anal. Mach. Intell., Vol. 25, No. 6, pp. 748-763, 2002.

[8] L. Sirovich and M. Kirby. "Low dimensional procedure for the characterization of human faces.” JOSA-A, Vol. 4, No. 3, pp. 519-524, 1987.

[9] J. Yang, D. Zhang, A. F. Frangi and J. Yang. "Two-dimensional PCA: A new approach to appearance-based face representation and recognition." IEEE Trans. Pattern Anal. Mach. Intell., Vol. 26, No. 1, pp. 131-137, 2004.

[10] C. Aguerrebere, G. Capdehourat, M. Delbracio, M. Mateu, A. Fern'andez and F. Lecumberry. "Aguar'a: An Improved Face Recognition Algorithm through Gabor Filter Adaptation.” Instituto de Ingenier' 1a El'ectrica de la Facultad de Ingenier'1a, Universidad de la Rep’ublica, Montevideo, Uruguay, 2012. 
[11] L. Wiskott, J. M. Fellous, N. Kruger and C. Von Der Malsburg. "Face recognition by elastic bunch graph matching." In L. C. Jain, U. Halici, I. Hayashi, and S. B. Lee, editors, Intelligent Biometric Techniques in Fingerprint and Face Recognition, CRC Press, chapter 11, pp. 355-396, 1999.

[12] D. S. Bolme. "Elastic bunch graph matching." Master's thesis, Colorado State University, Fort Collins, Colorado, USA, 2003.

[13] Qiong Wang, Jingyu Yang and Wankou Yang. "Face Detection using Rectangle Features and SVM." International Journal of Intelligent Technology, Vol. 1, No. 3, 2012.

[14] Kavita Shelke. "Face Recognition from Group Photograph." International Journal of Engineering and Innovative Technology (IJEIT), Vol. 3, Issue 1, 2013.

[15] V. Balamurugan, Mukundhan Srinivasan and Vijayanarayanan A. "A New Face Recognition Technique using Gabor Wavelet Transform with Back Propagation Neural Network." 2012.

[16] D. Sathish Kumar and S. Joshua Kumaresan. "REAL-TIME FACE RECOGNITION BASED ON OPTICAL FLOW AND HISTOGRAM EQUALIZATION." 2013.

[17] Chetna Singh, Sarvesh Singh and Prashant Baheti. "Human Face Recognition and Face Detection using Skin Colour Model.” International Journal of Current Engineering and Technology, Vol. 4, No. 4, 2014.

[18] V. Radha, N. Nallammal. "Neural Network Based Face Recognition Using RBFN Classifier." Proceedings of the World Congress on Engineering and Computer Science (WCECS), Vol. 1, 2011.

[19] Chunfei Ma, June-Young Jung, Seung-Wook Kim and Sung-Jea Ko. "Random projectionbased partial feature extraction for robust face recognition." Neurocomputing, Vol. 149, pp. 1232-1244, 2015.

[20] Suvigya Tripathi (09BEC094), Ankit V. Gupta, and Bhupendra Fataniya. "Face Recognition Technique." Review one, Dept. of Electronics and Communication Engineering, Nirma University at Ahmedabad, 2012.

[21] S. Ravindran S., S.C. Neoh and H. Muthusamy. "A hybrid expert system for automatic detection of voice disorders." Int. J. of Medical Engineering and Informatics, Vol. 6, No. 3, pp. $218-237,2014$.

[22] Z. Zeng, H. Zhang, R. Zhang and Y. Zhang. "A hybrid feature selection method based on rough conditional mutual information and Naive Bayesian classifier." ISRN Applied Mathematics, pp. 1-11, 2014.

[23] A.W. Mohemmed, M. Zhang and M. Johnston. "Particle swarm optimization based Adaboost for face detection." IEEE Congress on Evolutionary Computation, pp. 24942501, 2009. 
[24] N. L. Ajit Krisshna, V. Kadetotad Deepak, K. Manikantan and S. Ramachandran. "Face recognition using transform domain feature extraction and PSO-based feature selection." Applied Soft Computing, Vol. 22, pp. 141-161, 2014

[25] B. Xue, M. Zhang and W.N. Browne. "Particle swarm optimization for feature selection in classification: a multi-objective approach." IEEE Transactions on Cybernetics, Vol. 43, No. 6, pp. 1656-1671, 2013.

[26] C. Liu and H. Wechsler H. "Evolutionary pursuit and its application to face recognition." IEEE Transactions on Pattern Analysis and Machine Intelligence, Vol. 22, No. 6, pp. 570$582,2000$.

[27] A.P. Gosavi and S.R. Khot. "Facial expression recognition using principal component analysis." International Journal of Soft Computing and Engineering, Vol. 3, No. 4, pp. 258262, 2013.

[28] M. Kaur, R. Vashisht and N. Neeru. "Recognition of facial expressions with principal component analysis and singular value decomposition." International Journal of Computer Applications, Vol. 9, No. 12, pp. 36-40, 2010.

[29] L. Sirovich and M. Kirby. "Low-Dimensional Procedure for the Characterization of Human Faces.” Journal of the Optical Society of America, Vol. 4, pp. 519-524, 1987.

[30] M. Turk and A. Pentland. "Eigenfaces for Recognition." Journal of Cognitive Neuroscience, Vol. 3, No. 1, pp. 71-86, 1991.

[31] Müge Çarıçı, and Figen Özen. "A Face Recognition System Based on Eigenfaces Method.” Procedia Technology, Vol. 1, pp. 118-122, 2012.

[32] Yang, X. S. (2010). A New Metaheuristic Bat-Inspired Algorithm, in: Nature Inspired Cooperative Strategies for Optimization (NISCO 2010) (Eds. Cruz, C.; Gonz'alez, J. R.; Pelta, D. A.; Terrazas, G), Studies in Computational Intelligence Vol. 284, Springer Berlin, pp. 65-74.

[33] Colin, T. (2000). The Varienty of Life. Oxford University Press, Oxford.

[34] X.-S. Yang. (2011). Bat algorithm for multi-objective optimization. International Journal of Bio-Inspired Computation, vol. 3, no. 5, pp. 267-274. 